\title{
A novel approach to managing COVID-19 patients; results of lopinavir plus doxycycline cohort
}

\author{
Banu Cakir ${ }^{1}$ (D) \\ Received: 9 September 2020 / Accepted: 20 November 2020 / Published online: 7 January 2021 \\ (C) Springer-Verlag GmbH Germany, part of Springer Nature 2021
}

Cag et al. have recently published their work entitled "A novel approach to managing COVID-19 patients; results of lopinavir plus doxycycline cohort." We congratulate the authors for their precious efforts in handling COVID-19 patients working on a step-wise approach for the best benefit of the patients, while minimizing the hospital's workload. Given the absence of any specific treatment for COVID-19, various drug combinations have been in use in several countries, and any successful regimen/algorithm is of interest to clinicians worldwide. Unfortunately, the majority of treatment-related studies published so far are observational in nature, with potential for selection and information biases; confounding control is often insufficient due to missing data on several variables of interest; and inadequate discussion of intrinsic limitations of the studies may evoke confusion among clinicians. In this respect, the manuscript needs clarification on some issues and opens a floor for discussion on the context, effectiveness, and safety of home-based treatment for COVID-19 [1].

The title and the conclusion mainly focus on the results of a unique combination of lopinavir and doxycycline use in 161 PCR-positive hospitalized COVID-19 cases. We understand on reading that these 161 were members of a subgroup of 2043 "suspected" COVID-19 patients admitted to the emergency department of a specific hospital. The authors conducted a 3-step management plan for all 2043 (only 475 were positive in molecular tests) with doxycycline in different combinations: those isolated at home were given hydroxychloroquine plus doxycycline for 3-5 days, moderate-to-severe cases were hospitalized and treated with lopinavir plus doxycycline plus ceftriaxone for 5 days, and favipiravir was added for salvage therapy. The authors concluded that "home isolation of mild cases is an effective means

Banu Cakir

bcakir@ hacettepe.edu.tr; banucakir4@gmail.com

1 Department of Public Health, Hacettepe University Faculty of Medicine, Ankara, Turkey to manage the burden of disease, while lopinavir plus doxycycline is an alternative to current treatment regimens for COVID-19." These claims can mislead the readers as present$\mathrm{ed}$, and some issues need further clarification for robust interpretations [1].

The results (Table 1) focus mainly on PCR-positive moderate-to-severe COVID-19 patients treated with lopinavir plus doxycycline plus ceftriaxone for 5 days. There is no control group; thus, the effectiveness of this drug combination is not evident, other than a case-fatality rate of $12.4 \%(20 / 161)$, as a rough estimate of success for treatment of such patients. Sample size $(n=161)$ limits modeling to identify predictors of survival in this group, and analyses are simply based on personal and clinical characteristics of survivors versus deceased [1].

The authors defined a case as that "with an epidemiologic risk factor (not explained in the text) who had body temperature of $\geq 38^{\circ} \mathrm{C}$ and/or respiratory system symptoms which cannot be fully explained by any other condition or disease." Reportedly, the laboratory had ceased respiratory viral PCR panels and all COVID-19 PCR-negative patients were reportedly diagnosed as having viral respiratory tract infection of unknown etiology. Figure 1 reveals that all 2043 patients were treated as COVID-19, despite only $23 \%$ were PCR-positive. If so, it is important to present findings on clinical progress, recovery rate, and complications (if any) of PCR-negative cases, as well. Clarification is needed on how likely it is to claim all these patients as COVID-19 cases [1].

Figure 2 represents the number and results of PCR tests completed over time; the relative increase in test numbers with a rather consistent test-positivity was linked to the "ongoing fear in the population," with no details on variations over time in availability of testing, testing criteria, and/or the profiles of those tested. It needs further clarification on why the authors did not discuss the robustness of PCR-negativity in Figure 2, while treating all PCR-negative individuals with vogue clinical symptoms as COVID-19 [1]. 
Home isolation is presented in the manuscript as an effective means to manage the burden of disease (on hospitals). Despite collective evidence against effectiveness of hydroxychloroquine for SARS-CoV-2, including several randomized clinical trials such as SOLIDARITY and RECOVERY, some clinicians still insist on using it in COVID-19 treatment in hospital setting, claiming inconclusive evidence [2-5]. However, there is no justification for its use in home-setting; both the World Health Organization and the Federal Drug Agency clearly caution against use of hydroxychloroquine or chloroquine for COVID-19 outside of the hospital setting [3, 6]. Under these circumstances, it is inexplicable why the authors used hydroxychloroquine together with doxycycline for home-isolated cases (even for PCR-negatives). Neither safety nor ethical issues concerning hydroxychloroquine use for COVID-19 outside home is discussed in the manuscript. The authors' sharing of their personal experience with home-based treatment with regards to patient satisfaction, frequency/follow-up of adverse effects, and the improvement in patients' condition will be enlightening.

It is essential for clinicians in the front lines and public health decision makers to use the best available evidence for effective management of COVID-19 [6, 7]. The hectic, rapidly changing, and labor-intensive environment in COVID-19 pandemic might hinder clinicians' ability to follow updated literature and/or to critically appraise the manuscripts they read. Thus, editorial boards, reviewers, and authors have key roles in an ongoing pandemic in assuring that all publications include detailed information on assets and limitations of the studies established. Otherwise, inconsistencies in study designs, sample size, inclusion criteria, patients profile, and drugs/drug combinations used across studies would create more dilemma to the clinicians in taking therapeutic decisions or offering management strategies [2].

\section{Compliance with ethical standards}

Conflict of interest The author declares that there is no conflict of interest.
Ethical approval Not applicable.

Informed consent Not applicable.

\section{References}

1. Cag Y, Isten S, Isık-Goren B, Baysal NB, Bektas B, Selvi E, Ergen P, Aydin O, Ucisik AC, Y1lmaz-Karadag F, Caskurlu H, Akarsu Ayazoglu T, Kocoglu H, Uzman S, Nural-Pamukcu M, Arslan F, Bas G, Kalcigolu MT, Vahaboglu H (2020) A novel approach to managing COVID-19 patients; results of lopinavir plusc doxycycline cohort. Eur J Clin Microbiol Inf Dis. https://doi.org/10.1007/ s100096-020-04016-1

2. Chakraborty D, Debnanth F, Biswas S, Bhatta M, Hanguly S, Deb AK, Saha MK, Dutta S (2002) Exploring repurposing potential of existing drugs in the management of COVID-19 epidemic: a critical review. J Clin Med Res 12(8):463471 https://www.jocmr.org/index. php/JOCMR/article/view/4241. Accessed 30 Aug 2020

3. World Health Organization. Solidarity- clinical trial for COVID-19 treatments. https://www.who.int/emergencies/diseases/novelcoronavirus-2019/global-research-on-novel-coronavirus-2019-ncov/ solidarity-clinical-trial-for-covid-19-treatments. Accessed 30 Aug 2020

4. RECOVERY. Randomized Evaluation of COVID-19 therapy. Statement from the Chief Investigators of the Randomized Evaluation of COVID-19 Therapy (RECOVERY) Trial on hydroxychloroquine, 5 June 2020 https://www.recoverytrial.net/ files/hcq-recovery-statement-050620-final-002.pdf. Accessed 30 Aug 2020.

5. Pacheco-Barrios K, Fregni F (2020) Evidence-based decision making during COVID-19 pandemic. PPCR J 6(1):1-2. https://doi.org/ 10.21801/ppcrj2020.61.1

6. U.S. Food and Drug Administration (2020) FDA cautions against use of hydroxychloroquine or chloroquine for COVID-19 outside of the hospital setting or a clinical trial due to risk of heart rhythm problems. https://www.fda.gov/drugs/drug-safety-and-availability/ fda-cautions-against-use-hydroxychloroquine-or-chloroquinecovid-19-outside-hospital-setting-or. Accessed 30 Aug 2020

7. Deana C (2020) The COVID-19 pandemic: is our medicine still evidence-based? Ir J Med Sci. https://doi.org/10.1007/s11845-02002258-8

Publisher's note Springer Nature remains neutral with regard to jurisdictional claims in published maps and institutional affiliations. 